\title{
ESTUDO TEÓRICO COMPUTACIONAL DO HEXAFLUORETO DE ENXOFRE: UM GÁS DE EFEITO ESTUFA
}

\author{
J. L. SILVA ${ }^{1}$, J. V. FERREIRA ${ }^{2}$, E A. C. PAVÃO ${ }^{3}$ \\ Instituto Federal de Educação, Ciência e Tecnologia de Alagoas ${ }^{1,2}$, Universidade Federal de Pernambuco ${ }^{3}$ \\ joacyferreira@hotmail.com² \\ Submetido 29/04/2017 - Aceito 28/03/2020 \\ DOI: $10.15628 /$ holos. 2020.5910
}

\section{RESUMO}

O hexafluoreto de enxofre (SF6), bastante utilizado como isolante térmico e em equipamentos elétricos, é um gás totalmente fluorado, se tornando um composto perene na atmosfera. Por ser um bom absorvedor de radiação infravermelha, é considerado um gás de efeito estufa. Neste trabalho através de um estudo teórico computacional, usando a teoria do funcional da densidade
(DFT), determinamos geometria, energia de ligação e propriedades vibracionais da molécula de SF6. Os resultados mostraram que essa molécula tem grande potencial de aquecimento, com uma maior faixa de absorção de radiação infravermelho dentro da janela atmosférica.

PALAVRAS-CHAVE: Efeito estufa, hexafluoreto de enxofre, Teoria do Funcional da Densidade, Modos vibracionais

\section{COMPUTATIONAL THEORY STUDY OF SULFUR HEXAFLUORIDE: A GREENHOUSE EFFECT GAS}

\begin{abstract}
Sulfur hexafluoride (SF6), widely used as a thermal insulator and in electrical equipment, is a totally fluorinated gas, becoming a perennial compound in the atmosphere. Because it is a good absorber of infrared radiation, it is considered a greenhouse gas. In this work, through a theoretical computational study using the
\end{abstract}

density functional theory (DFT), we determine geometry, binding energy and vibrational properties of the SF6 molecule. The results showed that this molecule has a high warming potential, with a wide range of absorption of infrared radiation within the atmospheric window.

KEY-WORDS: Greenhouse effect, Sulfur hexafluoride, Density Functional Theory, Vibrational modes. 


\section{INTRODUÇÃO}

Do total da luz de todos os comprimentos de onda que alcança a Terra, cerca de $50 \%$ é absorvida em sua superfície. Um pouco mais de $20 \%$ dessa luz é absorvida pelas gotículas de água presentes no ar e moléculas de gases, e o restante volta para o espaço pela reflexão nas nuvens, partículas em suspensão, gelo, neve, areia e outros corpos refletores, sem ser absorvido (COLIN, 2011). A retenção de energia pelos gases estufa decorre de um mecanismo físico-químico. A absorção está associada à ressonância entre a frequência de vibração molecular e a da onda eletromagnética incidente (BELÉM et al., 2016). A luz é absorvida de forma praticamente total quando sua frequência quase se iguala à frequência do movimento interno de uma molécula. Cada molécula possui suas próprias frequências naturais de vibração, absorvendo ondas eletromagnéticas de frequências específicas e gerando um espectro de absorção característico (MISKOLCZI, 2010). Os fótons infravermelhos têm energia suficiente para promover transições entre níveis vibracionais da molécula (BERBERAN \& SANTOS, 2012). Uma condição para se observar um sinal correspondente a um modo vibracional num espectro de infravermelho é haver variação de momento dipolo associada à respectiva vibração (GONSALVES et al., 2005). Segundo OLIVEIRA (2009), a diferenciação dos modos vibracionais de uma molécula é feita entre aqueles limitados à variação dos comprimentos de ligação (estiramentos) e aquelas que envolvem a variação dos comprimentos de ângulos de ligação (deformação). O efeito estufa ocorre quando uma parte de radiação infravermelha do Sol (região entre $5 \mu \mathrm{m}$ e $100 \mu \mathrm{m}$ ) e a emitida pela superfície da Terra é absorvida por alguns gases que estão presentes na atmosfera (ROCHA et al., 2009). Este fenômeno é responsável por manter o planeta aquecido (OLIVEIRA, 2009 ; COLIN, 2011). Nos comprimentos de ondas de 8 a $13 \mu \mathrm{m}$, ocorre a passagem dessa radiação sem qualquer impedimento para o espaço. Esta porção do espectro é chamada de janela atmosférica, apresentando radiação eletromagnética com frequência na faixa de 800 a $1400 \mathrm{~cm}^{-1}$ (TOLENTINO et al., 1998; MEDEIROS, 2011).

Entre os gases atmosféricos, chamamos a atenção para o hexafluoreto de enxofre $\left(\mathrm{SF}_{6}\right)$ devido ao seu potencial de danos no ambiente. Apesar da quantidade de $\mathrm{SF}_{6}$ existente na atmosfera ser relativamente pequena, está entre os elementos mais danosos para o efeito estufa. Estima-se que seu ciclo de vida natural esteja entre 800 e 3.200 anos (JABIRI et. al., 2010). Absorve no infravermelho ao redor de 12,5 $\mathrm{m}$ (TOLENTINO et al., 1998). A liberação desse gás na atmosfera em grande escala levaria a um significativo aumento de vários graus na temperatura do Planeta. Seu potencial para o efeito estufa é 22.200 vezes mais poderoso que o $\mathrm{CO}_{2}$. Por ser totalmente fluorado, é um composto bastante estável e perene na atmosfera (OGIBOSK, 2013). No Brasil, a liberação de $\mathrm{SF}_{6}$ na atmosfera é da ordem de duas toneladas por ano. Como seu consumo tem crescido a uma taxa de cerca de $7 \%$ ao ano, seu impacto estufa futuro pode ser bastante significativo (TOLENTINO et al., 1998).

Através de um estudo teórico computacional, com o uso da teoria do funcional da densidade (DFT), este trabalho tem o objetivo de mostrar como a molécula de $\mathrm{SF}_{6}$ atua absorvendo e emitindo radiação infravermelha. Verificando como ocorrem as ligações químicas, suas vibrações e as energias absorvidas dentro de uma faixa de comprimento de onda, determinamos onde ocorrem os 
maiores picos de absorção de radiação. Esse estudo pretende contribuir para a compreensão em nível molecular do papel do $\mathrm{SF}_{6}$ para o aquecimento global.

\section{MÉTODO COMPUTACIONAL}

Os cálculos foram realizados usando DFT (VIANNA et al., 2004), com o funcional B3PW91 e o conjunto de funções de bases $6-31++G(d, p)$ como implementados no programa GAUSSIAN 2009. É um nível de cálculo com capacidade para reproduzir com qualidade dados experimentais de energias e distâncias de ligação da molécula.

\section{RESULTADOS E DISCUSSÃO}

Apresentaremos a seguir os resultados das propriedades eletrônicas calculadas: distância da ligação entre os átomos de enxofre e flúor, energia de ligação, frequências vibracionais e intensidade de energia absorvidas para a molécula de $\mathrm{SF}_{6}$. Comparamos nossos resultados com resultados experimentais e outros estudos teóricos disponíveis para a molécula de $\mathrm{SF}_{6}$.

\subsection{Otimização geométrica e Energia de ligação}

A primeira etapa do trabalho consistiu na otimização geométrica da molécula de SF6 no estado fundamental. A geometria obtida pode ser vista na Fig. 1.

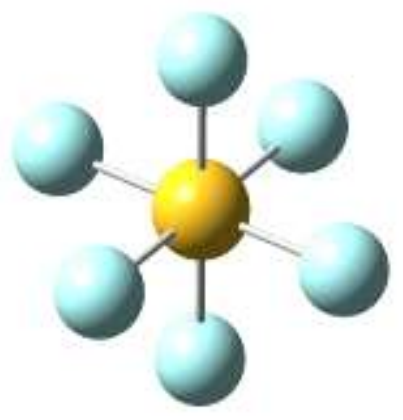

Figura 1. Estrutura molecular para o $\mathrm{SF}_{6}$

A Tabela 1 apresenta os resultados obtidos para a energia de ligação e distância da ligação (S-F) na molécula de SF6.

Tabela 1: Energia e distância de ligação calculados na molécula de $\mathrm{SF}_{6}$

\begin{tabular}{cccc}
\hline Parâmetros & Unidades & Teórico & Experimental \\
\hline ELIG & $\mathrm{eV}$ & 20,41 & 20,12 \\
$\mathrm{~d}(\mathrm{~S}-\mathrm{F})$ & $\AA$ & 1,599 & 1,564 \\
\hline
\end{tabular}

Os cálculos mostram que a molécula $\mathrm{SF}_{6}$ com sua geometria otimizada apresentou momento dipolo igual a zero no estado fundamental, ângulo de ligação F - S - F de $90^{\circ}$ e distâncias de ligações 
S-F de 1,599 ̊̊ em boa concordância com o valor experimental de 1,564 Å (CHRISTOPHOROU e OLTHOFF, 2000).

A energia de ligação ( $\left.E_{L I G}\right)$ foi obtida através da seguinte expressão:

$$
E_{L I G}=[E(S)+6 . E(F)]-E\left(S F_{6}\right)
$$

$E(S)$ é a energia total do átomo de enxofre, $E(F)$ a energia total do átomo de flúor e $E\left(S_{6}\right)$ a energia total da molécula de $\mathrm{SF}_{6}$. A energia de ligação calculada $(20,41 \mathrm{eV})$, se aproxima bem do valor encontrado experimentalmente $(20,12 \mathrm{eV})$. É bem melhor do que o valor obtido por BRUSKA E PIECHOTA $(26,81 \mathrm{eV})$, também através de um estudo DFT. A diferença do nosso trabalho está no funcional e no conjunto de funções de base de melhor qualidade que utilizamos.

\subsection{Frequências vibracionais}

Através da energia de absorção para todos os modos vibracionais do $\mathrm{SF}_{6}$, fora e dentro da janela atmosférica é possível identificar a contribuição desta molécula para o aquecimento global. Uma molécula não linear formada por $\mathrm{N}$ átomos possui (3N-6) modos de vibração (KUMIKO et al., 2011). Assim a molécula de $\mathrm{SF}_{6}$ possui 15 modos vibracionais. Na Tabela 2 relacionamos cada modo vibracional $(M V)$ da molécula com suas frequências vibracionais $(v)$, seguido de seus respectivos comprimentos de ondas $(\lambda)$ e a intensidade de absorção da radiação infravermelha (IR).

Tabela 2: Frequências vibracionais, Intensidade de absorção infravermelha e comprimentos de ondas calculados.

\begin{tabular}{|c|c|c|c|}
\hline MV & $\begin{array}{c}v \\
\left(\mathrm{~cm}^{-1}\right)\end{array}$ & $\begin{array}{c}\mathrm{IR} \\
\left(\mathrm{Km} \cdot \mathrm{mol}^{-1}\right)\end{array}$ & $\begin{array}{c}\lambda \\
(\mu \mathrm{m})\end{array}$ \\
\hline 1 & 316,67 & 0 & 31,58 \\
\hline 2 & 316,67 & 0 & 31,58 \\
\hline 3 & 316,67 & 0 & 31,58 \\
\hline 4 & 475,82 & 0 & 21,02 \\
\hline 5 & 475,82 & 0 & 21,02 \\
\hline 6 & 475,82 & 0 & 21,02 \\
\hline 7 & 554,68 & 64,84 & 18,03 \\
\hline 8 & 554,68 & 64,84 & 18,03 \\
\hline 9 & 554,68 & 64,84 & 18,03 \\
\hline 10 & 616,71 & 0 & 16,22 \\
\hline 11 & 616,71 & 0 & 16,22 \\
\hline 12 & 729,54 & 0 & 13,71 \\
\hline 13 & 883,47 & 630,71 & 11,32 \\
\hline
\end{tabular}




\begin{tabular}{llll}
14 & 883,47 & 630,71 & 11,32 \\
15 & 883,47 & 630,71 & 11,32 \\
\hline
\end{tabular}

Observa-se na Tabela 2 que dos 15 modos vibracionais existentes na molécula, os modos 1 , 2, 3, 4, 5, 6, 10, 11 e 12 apresentam intensidades de absorção nulas, ou seja, não absorvendo luz IV. Os modos vibracionais 7, 8 e 9 são os modos de vibração que apresentam as absorções iniciais de IV (infravermelha) fora do intervalo da janela atmosférica com frequência de $554,68 \mathrm{~cm}^{-1}$ e intensidade de $64,84 \mathrm{~km} \cdot \mathrm{mol}^{-1}$. Os modos vibracionais 13,14 e 15 vibram com as maiores frequências e intensidades, $883,47 \mathrm{~km} \cdot \mathrm{cm}^{-1}$ e $630,71 \mathrm{~km} \cdot \mathrm{mol}^{-1}$, respectivamente, e encontram-se inseridas na faixa de comprimento de onda correspondente à janela atmosférica $(11,32 \mu \mathrm{m})$. Esses modos absorvem as maiores quantidades de energia radiativa infravermelha térmica, sendo assim os responsáveis pelo maior potencial de aquecimento da molécula $\mathrm{SF}_{6}$ no efeito estufa, em concordância com BERA et al., 2009.

\subsection{Intensidades totais absorvidas de radiação infravermelha}

A figura 2 apresenta os valores de intensidades de radiação infravermelha absorvida pelo $\mathrm{SF}_{6}$. Verifica-se que a molécula absorve um valor total de energia igual a $2.086,65 \mathrm{~km} . \mathrm{mol}^{-1}$, sendo $1.892,12 \mathrm{~km} . \mathrm{mol}^{-1}$ absorvido dentro da faixa da janela atmosférica, correspondendo a uma fração de $93 \%$ de energia absorvida, mostrando que o $\mathrm{SF}_{6}$ é um gás estufa traço, mas com um grande potencial de aquecimento.

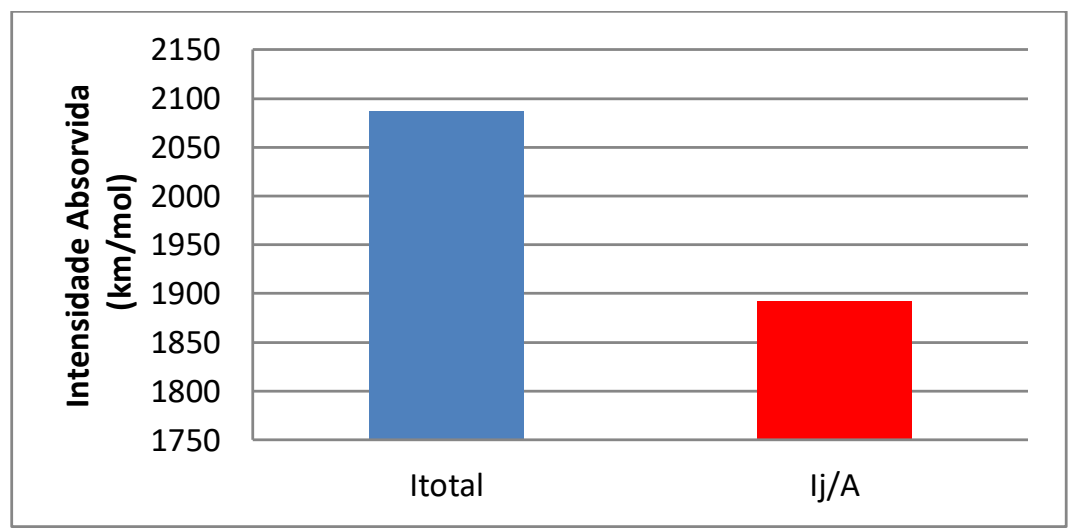

Figura 2. Valores de intensidades totais e absorvidas dentro da janela atmosférica.

\section{CONCLUSÃO}

Os cálculos DFT das propriedades vibracionais mostraram que $\mathrm{SF}_{6}$ é uma molécula que essencialmente absorve radiação infravermelha dentro da janela atmosférica. Como consequência, mesmo em pequena concentração na atmosfera, pode ter grande influência no aquecimento global. Daí a necessidade de um maior controle da emissão desse gás na atmosfera. 


\section{REFERÊNCIAS}

BARD, COLIN. (2011). Química ambiental, 4o ed.,Porto Alegre.

JULIO CESAR ROCHA, ANDRÉ HENRIQUE ROSA, ARNALDO ALVES CARDOSO. (2009). Introdução à Química Ambiental. 2a Edição.

GABRIELA BITTO DE OLIVEIRA, LARISSA FERNANDES BATISTA, PEDRO HENRIQUE PICELLI DE AZEVEDO. (2009). O efeito estufa. UNESP-FACULDADE DE CIÊNCIA E TECNOLOGIA.

TOLENTINO M. ; ROCHA-FILHO, R.C. (1998). A química no efeito estufa. Química Nova na Escola, n. 8.

FLÁVIO SOARES MEDEIROS.(2011). Assinatura Molecular do Aquecimento Global: um estudo teórico de aglomerados de moléculas de gás de efeito estufa. Universidade Federal do Amazonas.

BELÉM, F. R. L.; da Silva, D. W. A.; Sena Jr., D. M. (2016). Desenvolvimento de um Programa para Cálculo do Fator de Escala Vibracional. Rev. Virtual Quim. Data de publicação na Web: 9 de abril de 2016.

FERENC MISKOLCZI. (2010). Greenhouse Effect and the IR Radiative Structure of the Earth's Atmosphere. Int. J. Environ. Res. Public Health.

M.N. BERBERAN e SANTOS.(2012). Espectroscopia - princípios de química - física. Instituto superior técnico.

ANTÓNIO M. D' A. ROCHA GONSALVES, MARIA ELIZA DA SILVA SERRA, MARTA PIFIEIRO. (2005). Espectroscopia vibracional e eletrônica. Impressa da Universidade Coimbra.

JABIRI, Z. N.; Sharafi, D. (2010).Reducing SF6 emission from HV circuit breakers - A life cycle approach. In: Power and Energy Engineering Conference (APPEEC), Asia-Pacific. Anais mar. 2010.

LUCIANO OGIBOSKI. (2013). Extensão de ciclo de vida de uma subestação isolada a gás SF $_{6}$ por meio de um sistema de monitoramento de tempo real.Tese doutorado, São Paulo.

JOSÉDAVID M. VIANNA; SYLVIO CANUTO; ADALBERTO FAZZIO. (2004). Teoria Quântica de Moléculas e Sólidos - Simulação Computacional. Editora: Livraria da Física.

GAUSSIAN 09. (2009). REVISION A.1, M. J. FRISCH, G. W. TRUCKS, H. B. SCHLEGEL, G. E. SCUSERIA, M. A. ROBB, J. R. CHEESEMAN, G. SCALMANI, V. BARONE, B. MENNUCCI, G. A. PETERSSON, H. NAKATSUJI, M. CARICATO, X. LI, H. P. HRATCHIAN, A. F. IZMAYLOV, J. BLOINO, G. ZHENG, J. L. SONNENBERG, M. HADA, M. EHARA, K. TOYOTA, R. FUKUDA, J. HASEGAWA, M. ISHIDA, T. NAKAJIMA, Y. HONDA, O. KITAO, H. NAKAI, T. VREVEN, J. A. MONTGOMERY, JR., J. E. PERALTA, F. OGLIARO, M. BEARPARK, J. J. HEYD, E. BROTHERS, K. N. KUDIN, V. N. STAROVEROV, R. 
KOBAYASHI, J. NORMAND, K. RAGHAVACHARI, A. RENDELL, J. C. BURANT, S. S. IYENGAR, J. TOMASI, M. COSSI, N. REGA, J. M. MILLAM, M. KLENE, J. E. KNOX, J. B. CROSS, V. BAKKEN, C. ADAMO, J. JARAMILLO, R. GOMPERTS, R. E. STRATMANN, O. YAZYEV, A. J. AUSTIN, R. CAMMI, C. POMELLI, J. W. OCHTERSKI, R. L. MARTIN, K. MOROKUMA, V. G. ZAKRZEWSKI, G. A. VOTH, P. SALVADOR, J. J. DANNENBERG, S. DAPPRICH, A. D. DANIELS, O. FARKAS, J. B. FORESMAN, J. V. ORTIZ, J. CIOSLOWSKI, AND D. J. FOX, GAUSSIAN, INC., WALLINGFORD CT.

L.G. CHRISTOPHOROU, J.K. OLTHOFF, Electron interactions with SF 6 , J. Phys. Chem. Ref. Data 29 (2000), p. 267.

JACEK PIECHOTA, MARTA KINGA BRUSKA. (2008). Density Functional Study of Sulfur Hexauoride (SF6) and its Hydrogen Derivatives. Molecular Simulation, Journal of Experimental Nanoscience, Taylor \& Francis, 34 (10-15), pp.1041-1050.

SAKANE, K. K.; CARDOSO. M. A. G., BELTRAME JÚNIOR, M.; UEHARA, M. (2011). Espectroscopia Infravermelhos da Creatina. Revista Univap, São José dos Campos-SP, v. 17, n. 29, ago. 2011.

P. P. BERA, J. S. FRANCISCO and T. J. LEE. (2009). J. Phys. Chem. A , 113, 12694-12699. 